\title{
Age-related changes of neutrophil characteristics, potential of activation, DNA release and NET formation
}

Moritz Krall

Medizinische Universitat Wien

Lisa-Marie Mauracher

Medizinische Universitat Wien

Johanna Roiss

Medizinische Universitat Wien

Lena Hell

Medizinische Universitat Wien

Anna S Ondracek

Medizinische Universitat Wien

Johanna Gebhart

Medizinische Universitat Wien

Christine Brostjan

Medizinische Universitat Wien

Cihan Ay

Medizinische Universitat Wien

Ingrid Pabinger ( $\nabla$ ingrid.pabinger@meduniwien.ac.at )

Medizinische Universitat Wien

\section{Research}

Keywords: neutrophil subpopulations, neutrophil function, ageing, healthy individuals, neutrophil extracellular traps

Posted Date: October 14th, 2020

DOl: https://doi.org/10.21203/rs.3.rs-18855/v2

License: (c) (1) This work is licensed under a Creative Commons Attribution 4.0 International License.

Read Full License 


\section{Abstract}

Background: Neutrophils are a heterogeneous population of leukocytes, which can be subdivided into high and low density neutrophils (HDNs/LDNs). They are known to fight intruders with different mechanisms, including the release of neutrophil extracellular traps (NETs), which have also been associated with thrombosis. Risks of infection and thrombosis increase with age. Differences in neutrophil subpopulations and functionality have been shown in various disease states, but investigations in healthy subjects and their dependence on age are lacking. The aim of this study was to investigate age-related changes in neutrophils regarding neutrophil subpopulations, their potential of activation, DNA release and NET formation.

Methods: Neutrophil subpopulations (HDNs and LDNs) were isolated from 25 healthy individuals subdivided into 3 groups ( $<45$ years, $n=8 ; 45-54, n=9 ;>54, n=8$ ). Neutrophil characteristics, potential of activation and the ability of NET formation was investigated using flow cytometry. Externalisation of DNA was detected by a DNA release assay.

Results: HDN and LDN counts did not differ between age-groups. However, with increasing age we observed a shift in neutrophil subpopulations towards a lower amount of mature LDNs, characterized by their expression of membrane receptors $\mathrm{CD} 62 \mathrm{~L}$ and $\mathrm{CD} 16$. Upon stimulation, neutrophils of older individuals showed significantly higher release of DNA. HDNs of younger participants increased activation markers (CD66b and CD11b) to a higher extent compared to those of older individuals

Conclusion: Neutrophils and their ability of activation, DNA release and NET formation change with age and this might contribute to the higher risk of infection and thrombosis at advanced age. Furthermore, these results highlight the importance and necessity of age-matching in studies that focus on neutrophil characteristics.

\section{Background}

In healthy humans, between $50 \%$ and $70 \%$ of all circulating leukocytes are neutrophils. Originally thought to be homogeneous, neutrophils have meanwhile been shown to be a heterogeneous granulocyte population. It has been discovered that some neutrophils have lower density and appear within the peripheral blood mononuclear cell (PBMC) fraction when isolated. These so-called low density neutrophils (LDNs, often also referred to as low density granulocytes) were shown to promote tumour growth, immune suppression and angiogenesis (1). In healthy individuals, LDNs have been observed to account for 1 to $2 \%$ of the circulating neutrophils (2). High density neutrophils (HDNs, also called normal density neutrophils) are the majority of polymorphonuclear cells (PMNs) and have been described to exert protective anti-tumour properties (1). CD66b is consistently expressed by over $90 \%$ of neutrophils independent of their level of activation or state of the individual's health, making it a well-established marker of neutrophils. Together with CD11b (integrin alpha M), unique neutrophil characteristics and activation state can be determined $(3,4)$. 
Neutrophils have various features and responsibilities to fight invaders within a human's body, including phagocytosis, the release of reactive oxygen species as well as degranulation (5). In 2004, an additional defence mechanism, the so called neutrophil extracellular trap (NET) formation, was described (6). Upon neutrophil activation, peptidyl arginine deiminase 4 (PAD4) citrullinates histone H3 (H3Cit), which promotes chromatin decondensation and finally leads to the release of DNA into the extracellular surrounding. Negatively charged DNA as well as various neutrophil proteins contribute to trap and fight invaders and have also been described to be associated with thrombosis $(7,8)$.

While neutrophil ageing has been characterised before (9), the effects of the human ageing process on neutrophils, independent of neutrophil stage, has not been studied systematically. In 1991, Perskin and Cronstein (10) described that elderly people suffer higher rates of morbidity and mortality when confronted with infectious diseases compared to younger individuals. This is likely due to reduced functionality of immune cells of the adaptive and innate immune system $(11,12)$. The circulating neutrophil count is not altered in the elderly (13), but possibly neutrophil functions and recruitment to sites of inflammation are reduced, which may contribute to the vulnerability to infections (14). Neutrophil phagocytic capacity for opsonized bacteria and yeast has been described to be significantly decreased with age (15-17). Furthermore, reduced cytotoxicity (18-20) and increased circulating cytokine levels, which interfere with neutrophil function and recruitment $(18,21)$, were reported for the elderly.

Venous thromboembolism (VTE) is a major risk factor for morbidity and death in the general population, especially for patients with malignancy, hospitalisation and recent surgery (22). Generally, increasing age is one of the most relevant risk factors for VTE, as young individuals ( $<40$ years) have a low risk, individuals aged 40-60 years exhibit a moderate risk and older individuals ( $>60$ years) have a high risk (23). Neutrophils and NETs have been shown to be associated with thrombosis in cancer patients (7); however, the association between neutrophil subpopulations, activation of neutrophils and NET formation in the healthy and aging population is still unclear.

An important novel aspect that has very recently been brought into this discussion on the link between inflammation, age and thrombosis, where NETs most probably play a crucial role, is COVID-19. Middelton et al. argued that immunothrombosis triggered by NETs may be responsible for the prothrombotic state of COVID-19 patients and could therefore represent a promising target for therapeutic intervention. They observed that neutrophils from patients with COVID-19 are more prone towards NET release as compared to healthy individuals and that those patients develop severe thrombosis and respiratory failure (24).

We hypothesise that neutrophil characteristics, their potential of activation and their predisposition to undergo NET formation depends on the age of an individual. Our aim was to investigate age-related changes of LDNs and HDNs in healthy individuals to further understand immuno-regulatory functions and possible associations between increasing age and infectious and thrombotic risk.

\section{Results}

\section{Neutrophil subpopulations shift with increasing age}


Total neutrophil numbers were not statistically different in the various age groups (Table 1). In the investigation of quantitative changes of neutrophil subpopulations among age groups, cell counts of LDNs and HDNs were compared. LDN and HDN count did not show significant differences between the age groups (Figure 1A and B, Supplementary Table 1A and B). A significant difference was found in isolated mature (CD62 $\left.\mathrm{L}^{\text {high}} / \mathrm{CD} 16^{\text {high }}\right)$ LDNs with the biggest difference between the youngest and the oldest age group (Figure 1C). Age and mature LDNs, as continuous variables, were significantly correlated (spearman: $r^{2}=-0.468, p=0.018$, Table 2). Mature HDNs show a tendency towards lower numbers in the more advanced age groups; however, no statistically significant difference could be observed. No significant differences in the counts of activated (CD62 $\left.\mathrm{L}^{\text {low }} / \mathrm{CD} 16^{\text {high }}\right) \mathrm{HDNs}$ and LDNs were found between the age groups (Figure 1D). In Figure 1E a trend towards an increase of newly released (CD62 Ligh/CD16 low) HDNs and LDNs with age can be seen, albeit this difference was not statistically significant. Taken as continuous variable, age was positively correlated with newly released LDNs $\left(r^{2}=0.439, p=0.028\right.$, Table 2$)$. No differences in neutrophil count, subpopulations or other investigated parameters were found between male and female individuals (data not shown).

\section{CD66b and CD11b expression levels on HDNs and LDNs and its association with age}

CD66b expression of HDNs and LDNs showed a trend towards decrease with age (Figures 2A and 2B), whereas the statistical analysis between the groups revealed no significant difference. PMA stimulation revealed an association of age with a decreased ability to upregulate CD66b on the surface (100nM: $\mathrm{r}^{2}=$ $-0.430, p=0.032 ; 600 n M: r^{2}=-0.468, p=0.018$ ) (Figure 2A and B, Table 2, Supplementary Table 2A and B).

CD11b expression levels were significantly lower in HDNs of older donors upon stimulation with 10 and PMA compared to the youngest age group (Figure 2C, Supplementary Table 2C). Correlation analysis showed that there is again a negative correlation between upregulation of activation markers upon stimulation and increasing age $\left(1 \mu \mathrm{M} I 0: r^{2}=-0.430, p=0.032,4 \mu \mathrm{M} ; 10: r^{2}=-0.378, p=0.062 ; 100 n M\right.$ PMA:

$r^{2}=-0.480, p=0.015 ; 600 n M$ PMA: $r^{2}=-0.432, p=0.031 ;$ Table 2). No differences in CD11b expression levels were observed in LDNs (Figure 2D, Supplementary Table 2C and 2D).

\section{Increasing individuals' age and correlation with DNA release}

Stimulation with different concentrations of $\mathrm{IO}$ and PMA showed that PMNs from older individuals react more intensively, in terms of DNA externalisation, compared to cells from younger volunteers (Figure 3A, Supplementary Table 3A). This was especially seen after stimulation with higher doses of IO and lower doses of PMA. Furthermore, we correlated DNA release by PMNs with age and significant correlations were already observed in unstimulated samples $\left(r^{2}=0.559, p=0.004\right)$, which even increased with rising 10 concentrations ( $4 \mu \mathrm{M}: r^{2}=0.430, p=0.032 ; 6 \mu \mathrm{M}: r^{2}=0.515, p=0.008 ; 8 \mu \mathrm{M}: r^{2}=0.590, p=0.002 ;$ Table 2). DNA release after low dose PMA also correlated with age $\left(600 n M: r^{2}=0.545, p=0.005 ; 1 \mu \mathrm{M}: r^{2}=0.407, p=0.044\right.$; Table 2). 
A similar trend was seen in the PBMC fraction comprising the LDNs (Figure 3B, Supplementary Table 3B), although a statistical significance was only seen upon stimulation with $1 \mu \mathrm{M}$ PMA. Again, correlation analysis revealed significant age dependency $\left(1 \mu \mathrm{M}\right.$ PMA: $\mathrm{r}^{2}=0.458, p=0.021$; Table 2$)$.

\section{Ability of NET formation of HDNs and LDNs in various age groups}

$\mathrm{H} 3 \mathrm{Cit}$ intensity in HDNs at baseline and after stimulation for $3 \mathrm{~h}$ did not show differences between age groups (Figure 4A, Table 2, Supplementary Table 4A). Correlation with age as a metric variable revealed a positive significant association with $\mathrm{H} 3$ Cit intensity after stimulation with 600nM PMA $\left(r^{2}=0.458\right.$, $p=0.021)$. LDNs show a more pronounced difference between age groups. H3Cit intensity was significantly higher at baseline in individuals aged between 45 and 54 when compared to individuals younger than 45 (AG2 2.93-fold increase from AG1; Figure 4B, Supplementary Table 4B). Correlation analysis showed a positive association between increasing age and $\mathrm{H} 3$ Cit intensity in LDNs at baseline $\left(r^{2}=0.408, p=0.043\right)$. Similar results were observed in unstimulated as well as 10 stimulated samples (AG2 fold increase compared to AG1: baseline: 2.9-fold; unstimulated: 2.5-fold; $4 \mu \mathrm{M}$ IO: 1.79-fold; AG2 fold increase compared to AG3: baseline: 1.2-fold, unstimulated 1.7-fold, $4 \mu \mathrm{M}$ I0: 1.6-fold). The same trend was observed upon PMA stimulation, but this was not statistically significant (Figure 4B, Supplementary Table 4B).

\section{Discussion}

In this systematic investigation of neutrophils from healthy individuals of different age we could clearly demonstrate that neutrophil characteristics, potential of activation and ability to undergo NET formation change with human age. We observed a shift in neutrophil subpopulations towards decreased mature LDNs, but also lower ability to upregulate the activation marker CD11b upon stimulation with IO and PMA with increasing age. This may relate to altered neutrophil functions with increasing human age and contribute to the well-known higher susceptibility to pathogens in persons of advanced age. NET formation also correlated with age and was lowest in younger persons. The risk of thrombosis also increases with age and neutrophils of individuals at younger age might be less prone to prothrombotic actions.

Data on LDN frequency in healthy controls are rarely available and LDN counts vary between reports (25, 26). HDN and LDN counts showed no age related differences, however, percentages of mature $\mathrm{CD} 62 \mathrm{~L}^{\text {high }} / \mathrm{CD} 16^{\text {high }}$ LDNs correlated negatively and percentage of newly released CD $16^{\text {low }} \mathrm{LDNs}$ positively with age. Sagiv and colleagues observed that neutrophils, especially newly released neutrophils, are increased in patients with cancer and show a pro-tumorigenic phenotype (27). However, none of our healthy volunteers had active or a history of cancer. Additionally, newly released neutrophils are less potent in their pathogen defence (28) and might thus contribute to higher infection susceptibility of the elderly. The decreased potency of the immune system is furthermore associated with increased incidence of patients with cancer and metastasis (29). 
A negative association with age and decreased ability to upregulate CD66b upon PMA stimulation of HDNs was observed. Expression levels of the activation marker CD11b of HDNs were associated with age. Upregulation of CD11b expression on HDNs was found to be distinctively more prominent in younger individuals upon stimulation with different stimulants at various concentrations. Data on age-related $\mathrm{CD} 66 \mathrm{~b}$ expression levels in neutrophils have to our knowledge not been published to date. Data on agerelated analysis of $C D 11 b$, which is part of the integrin $a M \beta 2$ (CD11b/CD18) complex and crucial for neutrophil adhesion, are available, yet scarce. Furthermore, age-related expression data on CD11b and CD66b levels upon in vitro stimulation have not been published to date. Our results show that NET formation which represents a mode of neutrophil-mediated pathogen defence is less effectively activated in older individuals and may hence in part explain the higher infection rate within the older population.

Data on DNA release after $3 \mathrm{~h}$ stimulation at $37^{\circ} \mathrm{C}$ is likely to correspond to the release of NETs, although we did not measure any NET specific marker in this experiment. Upon low dose PMA stimulation, levels of released DNA were elevated in isolated PMNs with increasing individuals' age and also in isolated PBMCs harbouring the LDN fraction. HDNs within the PMN fraction were isolated with a median purity of $97.05 \%$ (IQR: 96.05-97.95), while LDNs within the PBMC fraction showed a median abundance of $1.95 \%$ (IQR: 1.30-2.95). Further purification of PBMCs via magnetic-bead sampling or other experimental purification steps would result in pure LDNs but would also pre-activate cells. Therefore, it has to be considered that LDNs could not be separately analysed and other cells within the PBMC layer may release their DNA as well. Since this method was not specific for measuring only NETs but all extracellular DNA, it can be assumed that distinct amounts of DNA originate from neutrophils but also from other cells such as eosinophils, mast cells, and monocyte/macrophages, which have all previously been described to also form extracellular traps in a process termed ETosis (28, 30-32). To precisely distinguish between different types of cells, a microscopic analysis approach with neutrophil- and NET-specific staining could be used. This however is rather challenging, as markers other than H3Cit to reliably identify NETs in humans are scarce. Approaches with antibodies against histone-DNA complexes, myeloperoxidase and neutrophil elastase (all in combination with DAPI or Hoechst) might be promising but are accompanied by major disadvantages (33). In mouse models, Ly6G is widely used to identify neutrophils (34). Other cell death mechanisms also lead to the release of cfDNA into the extracellular space, but programmed cell death mechanisms (such as apoptosis) may take longer periods of time as our measurement intervals of $3 \mathrm{~h}$ (35). Higher levels of DNA release upon increased individuals' age could be explained by the elevated number of immature, newly released neutrophils that were previously reported to release their DNA also spontaneously $(36,37)$, regardless of the stimulus or the respective concentrations.

While DNA release was high in AG2 as well as AG3 (frequently exhibiting comparable median levels, see Figure 2), the NET marker H3Cit was highest in the middle aged group of 45-54 years (in baseline, unstimulated and IO stimulated samples). Considering the lower level of H3Cit positive LDNs in AG3, it could be argued that older people have higher numbers of immature neutrophils that most likely have released their NETs spontaneously in circulation and therefore could not be activated again ex vivo (36, 37). Another potential explanation for this discrepancy is that neutrophils may change to another pathway of NET induction, without the generation of H3Cit. In vitro studies have shown that distinct 
activation pathways via NADPH oxidase (38), myeloperoxidase and neutrophil elastase and/or via mitochondrial ROS production (39) may lead to NET formation in the absence of H3Cit generation. Thus, measurements of DNA release (in particular of the highly pure PMN fraction) may more reliably and comprehensively reflect the NET forming capacity of isolated neutrophils than H3Cit levels. In our approach of investigating changes in $\mathrm{H} 3 \mathrm{C}$ it levels, flow cytometry was utilized to measure extracellular H3Cit. To gain further insights on intracellular H3Cit expression, microscopic analysis would be needed. Regarding the link of NET formation and venous thrombosis, which has been investigated before $(40,41)$, it is known that age is an important risk factor for VTE (42). One hypothesis is the increased blood coagulability (42). But also, the increased release of DNA by HDNs (or possibly also other cell types) may contribute to this increased risk. Recently it has additionally been highlighted, that in the context of COVID-19, neutrophils exhibit an increased tendency to release NETs. Since in their study the patient cohort was comprised of individuals aged $48.2 \pm 13.6$ (mean \pm SD) for non-ICU COVID-19 patients and $64.5 \pm 13.7$ (mean \pm SD) for ICU COVID-19 patients (24), it is an interesting research question whether neutrophils from persons with different age behave differently.

Some limitations of this study have to be addressed: the rather small number of participants, the chosen classification into age groups as well as the chosen stimuli. This healthy control cohort was designed to age and sex-match patients of other studies. The participants enrolled in this study were well defined with strict in- and exclusion criteria: healthy volunteers were excluded if they suffer from diseases that might impact neutrophil function, had a history of cancer, bleeding disorders, a history of thrombotic events or infectious diseases 6 weeks prior to the blood draw. The resulting healthy control cohort was then divided in three age groups of approximately even participant numbers. In this study, only chemical stimuli were used. PMA as well as IO are considered the "golden standard" in NET research, guaranteeing NET induction via different pathways and therefore reliable results. Since it was not our intent to investigate the effects of disease-associated NET inducers, but rather to see the effects of the individuals' age on NET forming capabilities when triggered via distinct pathways, reliability was prioritized. However, investigating the effects of biological stimuli under the same conditions could be an interesting approach to add further knowledge in the field of immunity and ageing. Also we did not cover exercise routine with the structured questionnaire, as it is known that exercise has many different effects on neutrophil numbers and behaviour. Regular exercise leads to an increase in endogenous pyrogen, a known neutrophil priming agent (43) and increases the numbers of circulating neutrophils (44). Lastly, LDN results have to be interpreted with caution, as there were fewer analysed events compared to HDNs. This study bears a number of strengths, one most important is that it is the first study that systematically analysed age related changes in neutrophils.

\section{Conclusion}

The human ageing process has a substantial impact on neutrophil subpopulations, activation potential as well as NET formation at baseline and upon stimulation. This study shows that there are age-related changes in neutrophils which potentially contribute to increased incidences of infections or thromboembolic events with increasing age. More studies are needed to clarify the functional impact in 
vivo. Our results further highlight the necessity of age-matching when comparing a healthy cohort to patients, especially but not limited to conditions with neutrophil involvement.

\section{Methods}

\section{Study population}

Between October 2018 and June 2019, 25 healthy adult individuals (median age [min-max]: 50 [25-64] years, female $\mathrm{n}=14$ ) were included in this study. Prior to a blood draw for routine parameters and experimental neutrophil analysis, all participants gave written informed consent and underwent a structured interview on their previous medical and bleeding history

From this cohort of healthy donors, three age groups were defined. Age group 1 (AG1) included volunteers younger than 45 years of age, age group 2 (AG2) included those between the age of 45 and 54 years and age group 3 (AG3) comprised those older than 54 years. Characteristics of the individuals in the different age groups are given in Table 1.

Written informed consent was given by each participant before inclusion. The study was conducted in conformity with the Declaration of Helsinki. Ethical approval for this study was given by the Ethics Committee of the Medical University of Vienna (EC-No 603/2009).

\section{Cell isolation}

Venous blood was collected in $9 \mathrm{~mL}$ K3EDTA blood tubes (VACUETTE®, Greiner Bio-One) and immediately layered over a density gradient for cell isolation (HISTOPAQUE®-1119; Sigma Aldrich and Ficoll-Paque $^{\text {TM }}$ PREMIUM 1077; GE Healthcare; $3 \mathrm{~mL}$ each). After 30 minutes of centrifugation (700 x g, no brakes, room temperature (RT)), the layer containing all peripheral blood mononuclear cells (PBMCs) as well as the LDNs and the layer containing all PMNs together with HDNs were isolated using a transfer pipette. Cells were washed with HBSS (Gibco) with an additional $1.26 \mathrm{mM} \mathrm{CaCl}_{2}$ (Merck; HBSS buffer; $800 \times \mathrm{g}, 5 \mathrm{~min}, \mathrm{RT}$ ). The supernatant was discarded and the pellet was resuspended in HBSS buffer. Cell counting was performed using a Sysmex XN-350 instrument (Sysmex).

Afterwards, cells for DNA release were diluted in HBSS buffer to final concentration of $10^{6}$ per $\mathrm{mL}$; cells for activation and H3Cit measurement were diluted in complete RPMI 1640 medium (with $10 \%$ fetal calf serum, penicillin $(100 \mathrm{U} / \mathrm{mL})$, streptomycin $(100 \mu \mathrm{g} / \mathrm{mL}), 20 \mathrm{mM}$ HEPES) to reach a final concentration of $5^{\star} 10^{5}$ cells $/ 45 \mu \mathrm{L}$. A portion of these cell suspensions was immediately fixed (referred to as baseline) with paraformaldehyde (PFA, final concentration: 1\%; Thermo Fisher).

Definition of analysed neutrophils

For both isolated PMN and PBMC cell fractions, samples were stained with a granulocyte-specific antibodiy (anti-CD66b; FITC, PB; BioLegend), anti-CD11b (APC; BioLegend) and anti-H3Cit (Abcam), either 
alone or in combination. This ensures that only granulocytes (HDNs within PMN, LDNs within PBMC) were analysed for expression levels of analysed markers.

\section{Subset analysis}

To investigate changes in neutrophil subsets, $5^{\star} 10^{5}$ immediately fixed cells were stained with anti-CD66b (1:5), anti-CD62L (AF647, 1:5; BioLegend), anti-CD16 (PE-Cy7, 1:5; BioLegend) and propidium iodide (PI, 1:5; BD Bioscience) for 15 minutes ( $\mathrm{min}$ ), RT, in the dark. Phosphate buffer saline (PBS, Gibco) was added to each sample to stop the staining procedure. All samples were analysed using a CytoFLEX S flow cytometer (Beckman Coulter). Single, live CD66+ cells were analysed according to their expression level of CD62L and CD16. Gates were defined in the following manner: Neutrophils with high CD62L and high CD16 expression were defined to be mature, low CD16 and high CD62L expression as newly released and cells low in CD62L, regardless of the CD16 expression levels, were defined as activated. Raw data was analysed using FlowJo V10 (FlowJo, LLC).

\section{Neutrophil activation}

Isolated neutrophils were activated for $30 \mathrm{~min}$ at $37^{\circ} \mathrm{C}$ with ionomycin (IO; final concentrations: $1 \mu \mathrm{M}$ and $4 \mu \mathrm{M}$; Thermo Fisher) and phorbol 12-myristate 13-acetate (PMA; final concentrations: 100nM and $600 \mathrm{nM}$; Sigma Aldrich). Afterwards, samples were fixed with paraformaldehyde (PFA; final concentration: $1 \%$, Thermo Fisher) and stained for $15 \mathrm{~min}, \mathrm{RT}$ in the dark with the following antibodies: anti-CD66b (1:4), anti-CD11b (1:4) and propidium iodide (PI, 1:4; BD Bioscience). PBS (Gibco) was added to each sample to stop the staining procedure. All samples were analysed using a CytoFLEX $S$ instrument (Beckman Coulter). For analysis, median fluorescent intensity (MFI) of CD66b and CD11b of live, single CD66b+ cells were investigated. Furthermore, "CD11b/CD66b high" neutrophils were defined by setting the respective immediately fixed samples as baseline. Data analysis was performed using FlowJo V10 (FlowJo, LLC).

\section{H3Cit measurement}

Stimulation of $10^{6}$ isolated cells in $90 \mu \mathrm{L}$ complete RPMI was performed using 10 (Thermo Fisher) and PMA (Sigma Aldrich), in the same concentrations as for neutrophil activation. After incubation for $3 \mathrm{~h}$ (and mixing every $30 \mathrm{~min}$ ) at $37^{\circ} \mathrm{C}$, samples were fixed with PFA (final concentration: 1\%; Thermo Fisher). Samples were blocked with PBS (Gibco) containing $2 \%$ bovine serum albumin (Sigma Aldrich; blocking buffer) first and then stained with a primary anti-H3Cit antibody (1:180) for 20 minutes at RT in the dark. After incubation, blocking buffer was added and samples were then centrifuged at $3200 \mathrm{x} \mathrm{g}$ for $10 \mathrm{~min}$ at $4^{\circ} \mathrm{C}$. Supernatant was discarded and secondary antibody (Alexa Fluor 647 goat anti-rabbit IgG, 1:10000; Invitrogen) in combination with anti-CD66b antibody (1:40) was added for 20 min in the dark at RT. After a further washing step using blocking buffer, PI (1:50; BD Bioscience) was added to all stained samples. One sample per cell fraction was left unstained; another was stained with only the secondary antibody to eliminated false positive signals due to unspecific antibody binding. As a gating strategy, dead CD66b+ singlets were analysed for their H3Cit expression. The gates for H3Cit+ cells were defined via the 
secondary only samples from the respective group. Samples were analysed using the CytoFLEX $S$ flow cytometer (Beckman Coulter $\left.{ }^{\circledR}\right)$ and FlowJo V10 (FlowJo, LLC).

\section{DNA release assay}

In this setup, $10^{5}$ cells per well were stimulated with 10 (final concentrations: $4 \mu \mathrm{M}, 6 \mu \mathrm{M}$ and $8 \mu \mathrm{M}$; Thermo Fisher) and PMA (final concentrations: $600 \mathrm{nM}, 1 \mu \mathrm{M}$ and $5 \mu \mathrm{M}$; Sigma Aldrich) in a FluoroNunc MaxiSorp 96-well plate (Thermo Fisher) for $2 \mathrm{~h} 45 \mathrm{~min}$ at $37^{\circ} \mathrm{C}$ in the dark (final volume per well: $300 \mu \mathrm{L}$ ). In addition to the stimulated samples, unstimulated cells (negative control) as well as Triton X-100-treated cells (which leads to complete cell lysis, thereby providing a positive control of $100 \%$ DNA release; final $0.33 \%$; Sigma Aldrich) were also included. Finally, SYTOX-Green (final concentration: $5 \mu \mathrm{M}$; Thermo Fisher) was added, incubated for $15 \mathrm{~min}$ at $37^{\circ} \mathrm{C}$ in the dark and samples were measured in triplicates with a multimode reader Varioskan LUX (ex.485/em.520; Thermo Fisher). All dilutions were prepared in HBSS buffer, all samples were measured in triplicates.

\section{Statistics}

Descriptive statistics were applied to describe the data. Categorical variables are given in frequencies and percentage, and continuous variables as median values and corresponding interquartile ranges (IQR). Kruskal-Wallis-test was performed to investigate differences between three groups. Statistical significance after Kruskal-Wallis is always depicted as dashed lines. Pairwise comparison was performed to investigate group to group differences, shown as solid lines. A two-sided p-value smaller than 0.05 was defined as significant. Statistical analysis was performed using SPSS version 17.0.2 (IBM). Graphs were generated with GraphPad Prism 6 (GraphPad).

\section{Abbreviations}

AG: Age group, HBSS: Hank's Balanced Salt Solution, HDNs: High density neutrophils, H3Cit: Citrullinated histone H3, IO: Ionomycin, IQR: Interquartile range, LDN: Low density neutrophils, MFI: Median fluorescent intensity, NETs: Neutrophil extracellular traps, PBMCs: Peripheral blood mononuclear cells, PBS: Phosphate buffered saline, PFA: Paraformaldehyde, PI: Propidium lodide, PMA: Phorbol 12myristate 13-acetate, PMNs: Polymorphonuclear cells, RT: Room temperature, VTE: Venous thromboembolism

\section{Declarations}

\section{Acknowledgements}

We thank the Core Facility Flow Cytometry, Medical University of Vienna for providing flow cytometric equipment and expertise.

\section{Authors' contributions}


MK, LMM and IP designed the study; MK, LMM, JR and LH designed and performed the experiments; LMM, JR, LH and JG recruited patients; MK, LMM, ASO, LH, CB, CA and IP analysed and interpreted the data; MK and LMM performed the statistical analyses. MK and LMM wrote the manuscript, which was reviewed, edited and finally approved by all other authors.

\section{Funding}

This work was funded by a grant from the Austrian Science Fund (FWF): Special Research Program "Inflammation and Thrombosis", SFB-F54.

\section{Availability of data and materials}

The datasets analysed during this study are available from the corresponding author on reasonable request.

\section{Ethics approval and consent to participate}

This study was conducted in conformity with the Declaration of Helsinki. Ethical approval was given by the Ethics Committee of the Medical University of Vienna (EC-No 603/2009).

\section{Consent for publication}

Each study participant gave written informed consent before inclusion.

\section{Competing Interests}

The authors have no conflict of interest to declare.

\section{Author details}

${ }^{1}$ Department of Medicine I: Clinical Division of Haematology and Haemostaseology, Medical University of Vienna, Vienna, Austria

2 Department of Internal Medicine II: Division of Cardiology, Medical University of Vienna, Vienna, Austria

3 Department of Surgery, Medical University of Vienna, Vienna, Austria

*These authors contributed equally to this work

\section{References}

1. Liu Y, Hu Y, Gu F, Liang J, Zeng Y, Hong X, et al. Phenotypic and clinical characterization of low density neutrophils in patients with advanced lung adenocarcinoma. Oncotarget. 2017;8(53):9096978. 
2. Yalavarthi S, Gould TJ, Rao AN, Mazza LF, Morris AE, Núñez-Álvarez C, et al. Antiphospholipid antibodies promote the release of neutrophil extracellular traps: a new mechanism of thrombosis in the antiphospholipid syndrome. Arthritis \& rheumatology (Hoboken, NJ). 2015;67(11):2990.

3. Lakschevitz FS, Hassanpour S, Rubin A, Fine N, Sun C, Glogauer M. Identification of neutrophil surface marker changes in health and inflammation using high-throughput screening flow cytometry. Experimental cell research. 2016;342(2):200-9.

4. Wright SD, Weitz JI, Huang AJ, Levin SM, Silverstein SC, Loike JD. Complement receptor type three (CD11b/CD18) of human polymorphonuclear leukocytes recognizes fibrinogen. Proceedings of the National Academy of Sciences. 1988;85(20):7734-8.

5. Mayadas TN, Cullere X, Lowell CA. The multifaceted functions of neutrophils. Annu Rev Pathol. 2014;9:181-218.

6. Brinkmann V, Reichard U, Goosmann C, Fauler B, Uhlemann Y, Weiss DS, et al. Neutrophil extracellular traps kill bacteria. Science. 2004;303(5663):1532-5.

7. Demers M, Krause DS, Schatzberg D, Martinod K, Voorhees JR, Fuchs TA, et al. Cancers predispose neutrophils to release extracellular DNA traps that contribute to cancer-associated thrombosis. Proc Natl Acad Sci U S A. 2012;109(32):13076-81.

8. Mauracher LM, Posch F, Martinod K, Grilz E, Daullary T, Hell L, et al. Citrullinated histone H3, a biomarker of neutrophil extracellular trap formation, predicts the risk of venous thromboembolism in cancer patients. J Thromb Haemost. 2018.

9. Adrover JM, Nicolas-Avila JA, Hidalgo A. Aging: A Temporal Dimension for Neutrophils. Trends Immunol. 2016;37(5):334-45.

10. Perskin MH, Cronstein BN. Age-related changes in neutrophil structure and function. Mech Ageing Dev. 1992;64(3):303-13.

11. Miller RA. The aging immune system: primer and prospectus. Science. 1996;273(5271):70-4.

12. Lord JM, Butcher S, Killampali V, Lascelles D, Salmon M. Neutrophil ageing and immunesenescence. Mech Ageing Dev. 2001;122(14):1521-35.

13. Born J, Uthgenannt D, Dodt C, Nunninghoff D, Ringvolt E, Wagner T, et al. Cytokine production and lymphocyte subpopulations in aged humans. An assessment during nocturnal sleep. Mech Ageing Dev. 1995;84(2):113-26.

14. Butcher S, Chahel H, Lord JM. Ageing and the neutrophil: no appetite for killing? Immunology. 2000;100(4):411-6.

15. Mege JL, Capo C, Michel B, Gastaut JL, Bongrand P. Phagocytic cell function in aged subjects. Neurobiol Aging. 1988;9(2):217-20.

16. Emanuelli G, Lanzio M, Anfossi T, Romano S, Anfossi G, Calcamuggi G. Influence of age on polymorphonuclear leukocytes in vitro: phagocytic activity in healthy human subjects. Gerontology. 1986;32(6):308-16. 
17. Wenisch C, Patruta S, Daxböck F, Krause R, Hörl W. Effect of age on human neutrophil function. Journal of leukocyte biology. 2000;67(1):40-5.

18. Esparza B, Sanchez H, Ruiz M, Barranquero M, Sabino E, Merino F. Neutrophil function in elderly persons assessed by flow cytometry. Immunol Invest. 1996;25(3):185-90.

19. Corberand J, Ngyen F, Laharrague P, Fontanilles AM, Gleyzes B, Gyrard E, et al. Polymorphonuclear functions and aging in humans. J Am Geriatr Soc. 1981;29(9):391-7.

20. Fülöp Jr T, Foris G, Worum I, Leövey A. Age-dependent alterations of Fc gamma receptor-mediated effector functions of human polymorphonuclear leucocytes. Clinical and experimental immunology. 1985;61(2):425.

21. MacGregor RR, Shalit M. Neutrophil function in healthy elderly subjects. Journal of gerontology. 1990;45(2):M55-M60.

22. Thrombosis: a major contributor to global disease burden. Thromb Res. 2014;134(5):931-8.

23. Blann AD, Lip GY. Venous thromboembolism. BMJ (Clinical research ed). 2006;332(7535):215-9.

24. Middleton EA, He XY, Denorme F, Campbell RA, Ng D, Salvatore SP, et al. Neutrophil extracellular traps contribute to immunothrombosis in COVID-19 acute respiratory distress syndrome. Blood. 2020;136(10):1169-79.

25. Ostendorf L, Mothes R, van Koppen S, Lindquist RL, Bellmann-Strobl J, Asseyer S, et al. Low-Density Granulocytes Are a Novel Immunopathological Feature in Both Multiple Sclerosis and Neuromyelitis Optica Spectrum Disorder. Front Immunol. 2019;10:2725.

26. Cloke T, Munder M, Taylor G, Muller I, Kropf P. Characterization of a novel population of low-density granulocytes associated with disease severity in HIV-1 infection. PLoS One. 2012;7(11):e48939.

27. Sagiv JY, Michaeli J, Assi S, Mishalian I, Kisos H, Levy L, et al. Phenotypic diversity and plasticity in circulating neutrophil subpopulations in cancer. Cell reports. 2015;10(4):562-73.

28. Pillay J, Ramakers BP, Kamp VM, Loi AL, Lam SW, Hietbrink F, et al. Functional heterogeneity and differential priming of circulating neutrophils in human experimental endotoxemia. J Leukoc Biol. 2010;88(1):211-20.

29. Palmer $S$, Albergante $L$, Blackburn CC, Newman TJ. Thymic involution and rising disease incidence with age. Proc Natl Acad Sci U S A. 2018;115(8):1883-8.

30. Guimaraes-Costa AB, Nascimento MT, Wardini AB, Pinto-da-Silva LH, Saraiva EM. ETosis: a microbicidal mechanism beyond cell death. Journal of parasitology research. 2012;2012.

31. von Köckritz-Blickwede M, Goldmann O, Thulin P, Heinemann K, Norrby-Teglund A, Rohde M, et al. Phagocytosis-independent antimicrobial activity of mast cells by means of extracellular trap formation. Blood. 2008;111(6):3070-80.

32. Yousefi S, Gold JA, Andina N, Lee JJ, Kelly AM, Kozlowski E, et al. Catapult-like release of mitochondrial DNA by eosinophils contributes to antibacterial defense. Nat Med. 2008;14(9):949-53.

33. de Buhr N, von Köckritz-Blickwede M. How Neutrophil Extracellular Traps Become Visible. Journal of immunology research. 2016;2016:4604713. 
34. Stackowicz J, Jönsson F, Reber LL. Mouse Models and Tools for the in vivo Study of Neutrophils. Front Immunol. 2019;10:3130.

35. Düssmann H, Rehm M, Kögel D, Prehn JH. Outer mitochondrial membrane permeabilization during apoptosis triggers caspase-independent mitochondrial and caspase-dependent plasma membrane potential depolarization: a single-cell analysis. J Cell Sci. 2003;116(Pt 3):525-36.

36. Lin AM, Rubin CJ, Khandpur R, Wang JY, Riblett M, Yalavarthi S, et al. Mast cells and neutrophils release IL-17 through extracellular trap formation in psoriasis. The Journal of Immunology. 2011;187(1):490-500.

37. Ortmann W, Kolaczkowska E. Age is the work of art? Impact of neutrophil and organism age on neutrophil extracellular trap formation. Cell and tissue research. 2018;371(3):473-88.

38. Papayannopoulos V, Metzler KD, Hakkim A, Zychlinsky A. Neutrophil elastase and myeloperoxidase regulate the formation of neutrophil extracellular traps. J Cell Biol. 2010;191(3):677-91.

39. Simon HU, Haj-Yehia A, Levi-Schaffer F. Role of reactive oxygen species (ROS) in apoptosis induction. Apoptosis : an international journal on programmed cell death. 2000;5(5):415-8.

40. Fuchs TA, Brill A, Wagner DD. Neutrophil extracellular trap (NET) impact on deep vein thrombosis. Arteriosclerosis, thrombosis, and vascular biology. 2012;32(8):1777-83.

41. Fuchs TA, Brill A, Duerschmied D, Schatzberg D, Monestier M, Myers DD, et al. Extracellular DNA traps promote thrombosis. Proceedings of the National Academy of Sciences. 2010;107(36):15880-5.

42. Crous-Bou M, Harrington LB, Kabrhel C. Environmental and Genetic Risk Factors Associated with Venous Thromboembolism. Semin Thromb Hemost. 2016;42(8):808-20.

43. Cannon JG, Kluger MJ. Endogenous pyrogen activity in human plasma after exercise. Science. 1983;220(4597):617-9.

44. Pyne DB. Regulation of neutrophil function during exercise. Sports Med. 1994;17(4):245-58.

\section{Tables}

Table 1. Characteristics of study participants. 


\begin{tabular}{|c|c|c|c|c|}
\hline$\underline{\text { Characteristics }}$ & $\begin{array}{l}\text { AG } 1 \\
(\leq 45) .\end{array}$ & $\frac{\underline{\mathrm{AG} \mathrm{2}}}{(\underline{45-54)})}$ & $\begin{array}{l}\underline{\mathrm{AG} 3} \\
(\geq 54)\end{array}$ & p-value \\
\hline Number of participants, $\underline{\mathrm{n}}$ & $\underline{8}$ & $\underline{9}$ & $\underline{8}$ & - \\
\hline Age, ,years; median (min-max). & $\begin{array}{c}\underline{33} \\
(\underline{25-40}) .\end{array}$ & $\begin{array}{c}\underline{50} \\
(\underline{48-52}) .\end{array}$ & $\begin{array}{c}\underline{61} \\
(\underline{55-64}) .\end{array}$ & - \\
\hline Female, $\%$ & $\underline{50}$ & $\underline{66}$ & $\underline{50}$ & - \\
\hline Leukocytes $[\underline{10} \underline{\underline{9}} / \mathrm{L}]$, median $(\underline{\mathrm{IQR}})$ & $\begin{array}{c}\underline{4.67} \\
(\underline{4.51-5.38)})\end{array}$ & $\begin{array}{c}\underline{6.41} \\
(\underline{4.88-8.12)})\end{array}$ & $\begin{array}{c}\underline{5.06} \\
(\underline{4.39-5.81)})\end{array}$ & $\underline{0.026}$ \\
\hline Neutrophils $[\underline{10} \underline{9} / \mathrm{L}]$, median (IQR). & $\begin{array}{c}\underline{2.8} \\
(\underline{1.9-2.9)} .\end{array}$ & $\begin{array}{c}\underline{3.5} \\
(\underline{2.75-5.25)})\end{array}$ & $\frac{\underline{2.75}}{(\underline{2.25-3.25)})}$ & $\underline{0.127}$ \\
\hline
\end{tabular}

Abbreviations: AG - age group, $\mathrm{n}$ - number of occurrences, y - years, IQR - interquartile range. Statistical analysis using Kruskal-Wallis test.

Table 2. Correlations with age as a continuous variable 
Information about correlations of variables shown in figure 1: Neutrophil characterisation

\begin{tabular}{ll|cc} 
& & $\mathrm{r}$ (spearman) & $\mathrm{p}$-value \\
\hline 1A & HDN count/mL & 0.040 & 0.849 \\
\hline 1B & LDN count/mL & 0.127 & 0.545 \\
\hline 1C & mature HDNs & -0.332 & 0.105 \\
& mature LDNs & -0.468 & 0.018 \\
\hline 1D & activated HDNs & -0.131 & 0.533 \\
& activated LDNs & -0.198 & 0.344 \\
\hline 1E & newly released HDNs & 0.395 & 0.050 \\
& newly released LDNs & 0.439 & $\mathbf{0 . 0 2 8}$
\end{tabular}

Information about correlations of variables shown in figure 2: CD66b and CD11b expression levels of HDNs and LDNs

\begin{tabular}{|c|c|c|c|}
\hline & & r (spearman) & p-value \\
\hline \multirow{6}{*}{$2 \mathrm{~A}$} & HDN CD66b baseline & -0.259 & 0.211 \\
\hline & HDN CD66b unstimulated & -0.149 & 0.476 \\
\hline & HDN CD66b $1 \mu \mathrm{M}$ IO & -0.120 & 0.568 \\
\hline & HDN CD66b $4 \mu \mathrm{M}$ IO & -0.052 & 0.805 \\
\hline & HDN CD66b 100 nM PMA & -0.43 & 0.032 \\
\hline & HDN CD66b 600 nM PMA & -0.468 & 0.018 \\
\hline \multirow[t]{6}{*}{$2 \mathrm{~B}$} & LDN CD66b baseline & 0.030 & 0.887 \\
\hline & LDN CD66b unstimulated & 0.236 & 0.256 \\
\hline & LDN CD66b $1 \mu \mathrm{M}$ IO & -0.214 & 0.305 \\
\hline & LDN CD66b $4 \mu \mathrm{M}$ IO & -0.059 & 0.780 \\
\hline & LDN CD66b 100 nM PMA & 0.091 & 0.666 \\
\hline & LDN CD66b 600 nM PMA & -0.039 & 0.852 \\
\hline \multirow[t]{6}{*}{$2 \mathrm{C}$} & HDN CD11b baseline & 0.282 & 0.172 \\
\hline & HDN CD1 $1 \mathrm{~b}$ unstimulated & 0.345 & 0.092 \\
\hline & HDN CD11b $1 \mu \mathrm{M}$ IO & -0.43 & 0.032 \\
\hline & HDN CD11b $4 \mu \mathrm{M}$ IO & -0.378 & 0.062 \\
\hline & HDN CD11b 100 nM PMA & -0.48 & 0.015 \\
\hline & HDN CD11b 600 nM PMA & -0.432 & 0.031 \\
\hline \multirow[t]{6}{*}{$2 \mathrm{D}$} & LDN CD11b baseline & -0.098 & 0.642 \\
\hline & LDN CD11b unstimulated & -0.191 & 0.361 \\
\hline & LDN CD11b $1 \mu \mathrm{M}$ IO & -0.354 & 0.083 \\
\hline & LDN CD11b $4 \mu \mathrm{M}$ IO & -0.185 & 0.375 \\
\hline & LDN CD11b 100 nM PMA & -0.121 & 0.565 \\
\hline & LDN CD11b 600 nM PMA & -0.350 & 0.086 \\
\hline
\end{tabular}

Information about correlations of variables shown in figure 3: DNA-release of PMNs and PBMCs

\begin{tabular}{ll}
\hline 3A & PMN unstimulated \\
& PMN $4 \mu M$ IO \\
PMN $6 \mu \mathrm{M}$ IO \\
PMN $8 \mu \mathrm{M}$ IO \\
PMN $0.6 \mu \mathrm{M}$ PMA
\end{tabular}

\begin{tabular}{l|cc} 
& $\mathrm{r}$ (spearman) & $\mathrm{p}$-value \\
\hline & 0.559 & 0.004 \\
0.43 & 0.032 \\
0.515 & 0.008 \\
0.59 & 0.002 \\
& 0.545 & 0.005
\end{tabular}




\begin{tabular}{|c|c|c|}
\hline $\begin{array}{l}\text { PMN } 1 \mu \mathrm{M} \text { PMA } \\
\text { PMN } 5 \mu \mathrm{M} \text { PMA }\end{array}$ & $\begin{array}{l}0.407 \\
0.346\end{array}$ & $\begin{array}{l}0.044 \\
0.090\end{array}$ \\
\hline PBMC unstimulated & 0.117 & 0.578 \\
\hline PBMC $4 \mu \mathrm{M}$ IO & 0.072 & 0.734 \\
\hline PBMC $6 \mu \mathrm{M}$ IO & 0.152 & 0.470 \\
\hline PBMC $8 \mu \mathrm{M}$ IO & 0.205 & 0.327 \\
\hline PBMC $0.6 \mu \mathrm{M}$ PMA & 0.371 & 0.068 \\
\hline PBMC $1 \mu \mathrm{M}$ PMA & 0.458 & 0.021 \\
\hline PBMC $5 \mu \mathrm{M}$ PMA & 0.106 & 0.614 \\
\hline
\end{tabular}

Information about correlations of variables shown in figure 4: Levels of H3Cit in $3 \mathrm{~h}$ stimulated HDNs and LDNs

\begin{tabular}{|c|c|c|c|}
\hline & & r (spearman) & p-value \\
\hline \multirow[t]{6}{*}{$4 \mathrm{~A}$} & HDN H3Cit baseline & 0.031 & 0.884 \\
\hline & HDN H3Cit unstimulated & -0.075 & 0.720 \\
\hline & HDN H3Cit $1 \mu \mathrm{M}$ IO & -0.078 & 0.712 \\
\hline & HDN H3Cit $4 \mu \mathrm{M}$ IO & -0.060 & 0.776 \\
\hline & HDN H3Cit 100nM PMA & 0.378 & 0.062 \\
\hline & HDN H3Cit 600nM PMA & 0.458 & 0.021 \\
\hline \multirow[t]{6}{*}{$4 \mathrm{~B}$} & LDN H3Cit baseline & 0.408 & 0.043 \\
\hline & LDN H3Cit unstimulated & 0.275 & 0.183 \\
\hline & LDN H3Cit $1 \mu \mathrm{M}$ IO & 0.295 & 0.153 \\
\hline & LDN H3Cit $4 \mu \mathrm{M}$ IO & -0.008 & 0.971 \\
\hline & LDN H3Cit 100nM PMA & 0.071 & 0.737 \\
\hline & LDN H3Cit 600nM PMA & 0.088 & 0.674 \\
\hline
\end{tabular}

Abbreviations: HDN - high density neutrophil, LDN - low density neutrophil, PMN polymorphonuclear cells, PBMC - peripheral blood mononuclear cell, IO - ionomycin, PMA phorbol 12-myristate 13-acetate

\section{Figures}




\section{Figure 1}

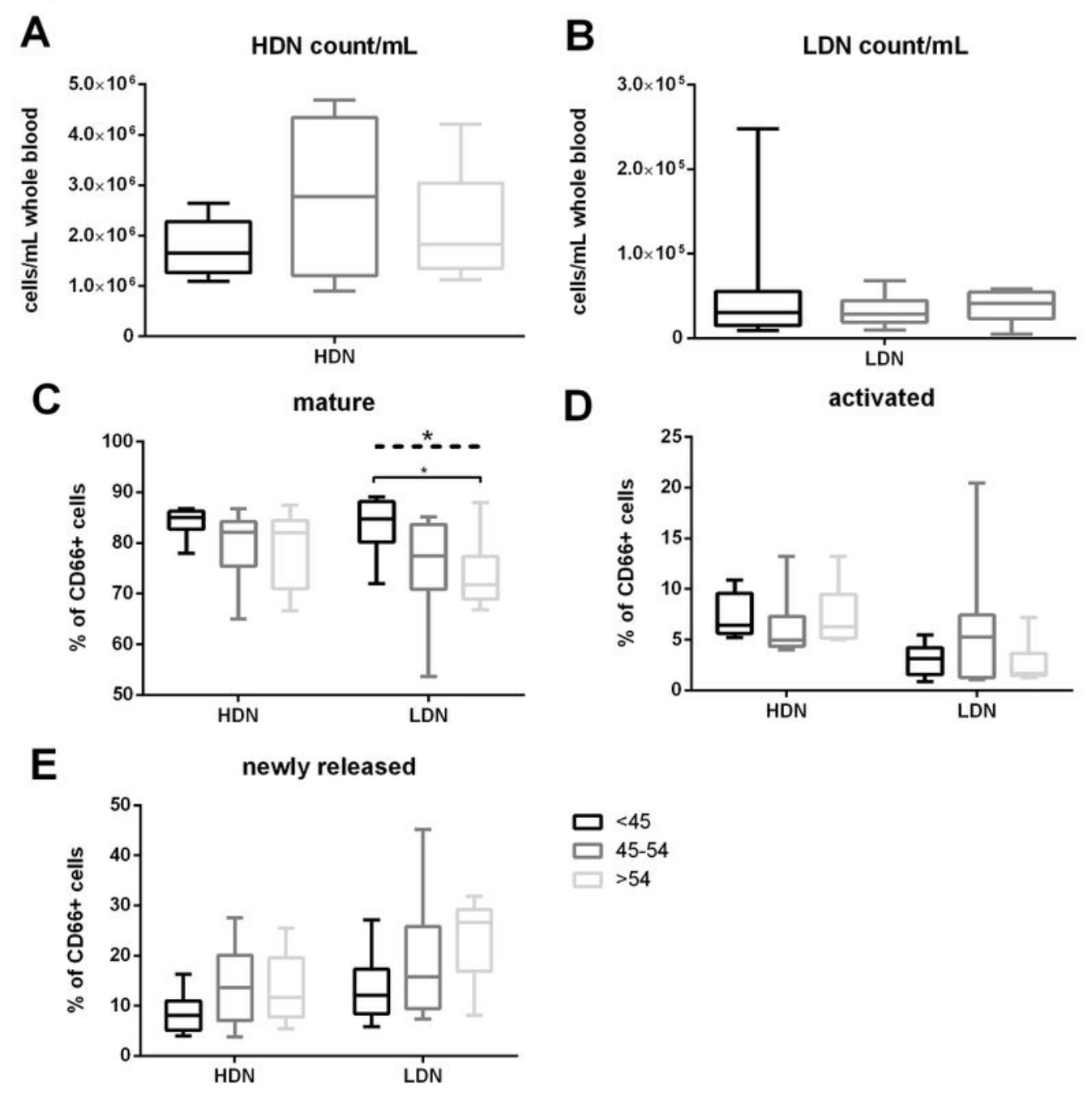

\section{Figure 1}

Neutrophil subpopulations. Box plots showing absolute numbers of isolated (A) high density neutrophils (HDN) and (B) low density neutrophils (LDN) per $\mathrm{mL}$ whole blood for all three age groups. (C) Percentage of CD66b positive mature HDN and LDN, distinguished from other neutrophils by their high expression of both CD62L and CD16 (CD62Lhigh/CD16high). (D) Percentages of CD66b positive, activated HDN and LDN, distinguished from other neutrophils by their low expression of CD62L and high expression levels of 
CD16 (CD62Llow/CD16high). (E) Percentages of CD66b positive, newly released HDN and LDN, distinguished from other neutrophil subsets by their low expression levels of CD16 (CD62Lhigh/CD16low). p-values (Kruskal-Wallis test in dashed lines; post hoc test in solid lines): * $\mathrm{p} \leq$ 0.05

\section{Figure 2}

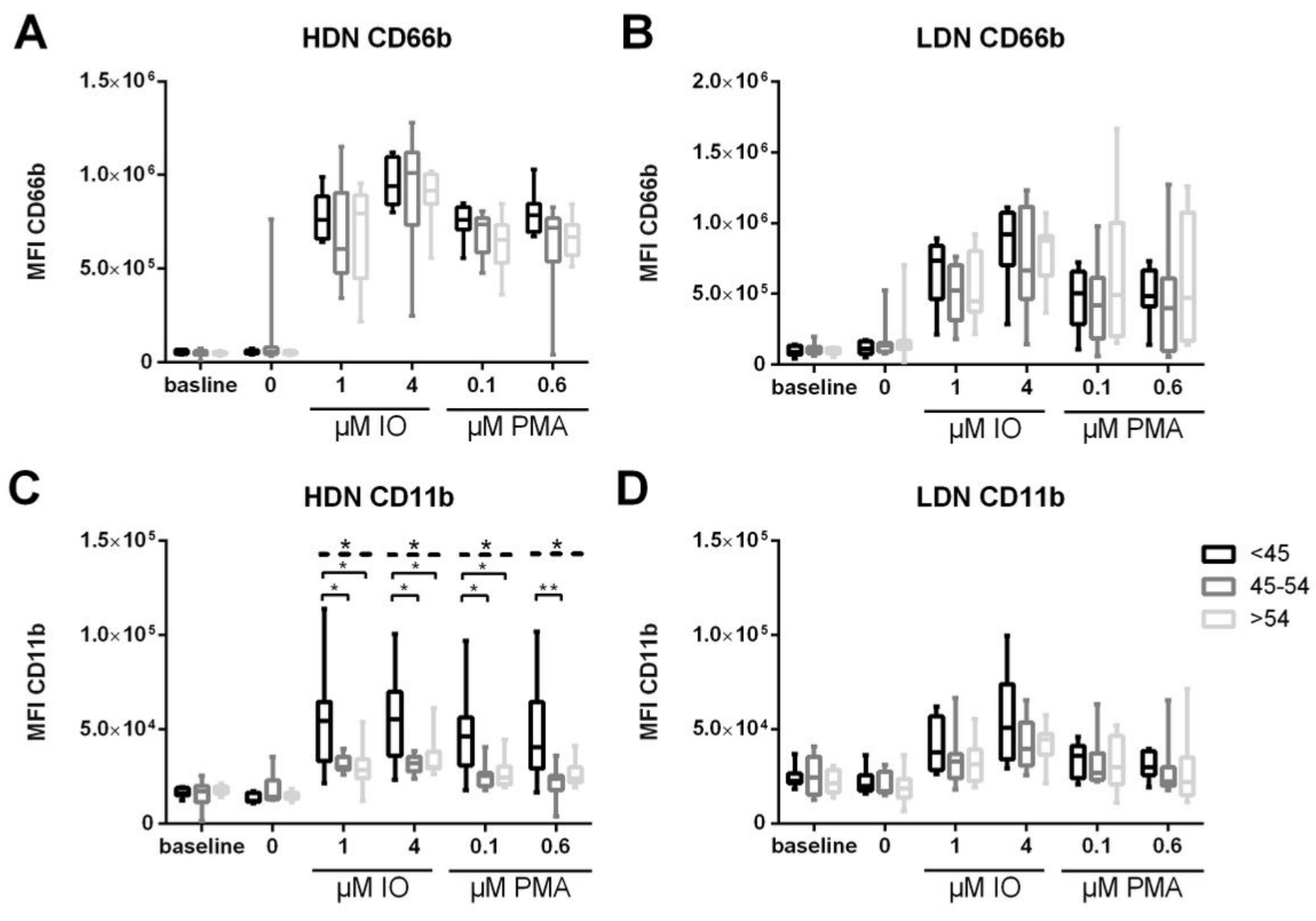

Figure 2

CD66b and CD11b expression levels of HDNs and LDNs. (A) Median fluorescence intensity (MFI) of CD66b positive, baseline, unstimulated $(0)$ as well as ionomycin $\left(10,3 \mathrm{~h} / 37^{\circ} \mathrm{C}\right)$ and phorbol 12 -myristate 13-acetate (PMA; $30 \mathrm{~min} / 37^{\circ} \mathrm{C}$ ) treated HDNs and (B) LDNs was measured. (C) MFI of CD11b unstimulated $(0)$ cells of the middle age group (45-54) showed increased CD11b expression, whereas stimulation with $\mathrm{IO}$ and PMA both showed an age-dependent decrease in CD11b positivity. (D) MFI of CD11b positive baseline, unstimulated $(0)$ as well as IO and PMA treated LDNs. p-values (Kruskal-Wallis test in dashed lines; post hoc test in solid lines): ${ }^{*} \leq 0.05, * * p \leq 0.01$ 
Figure 3

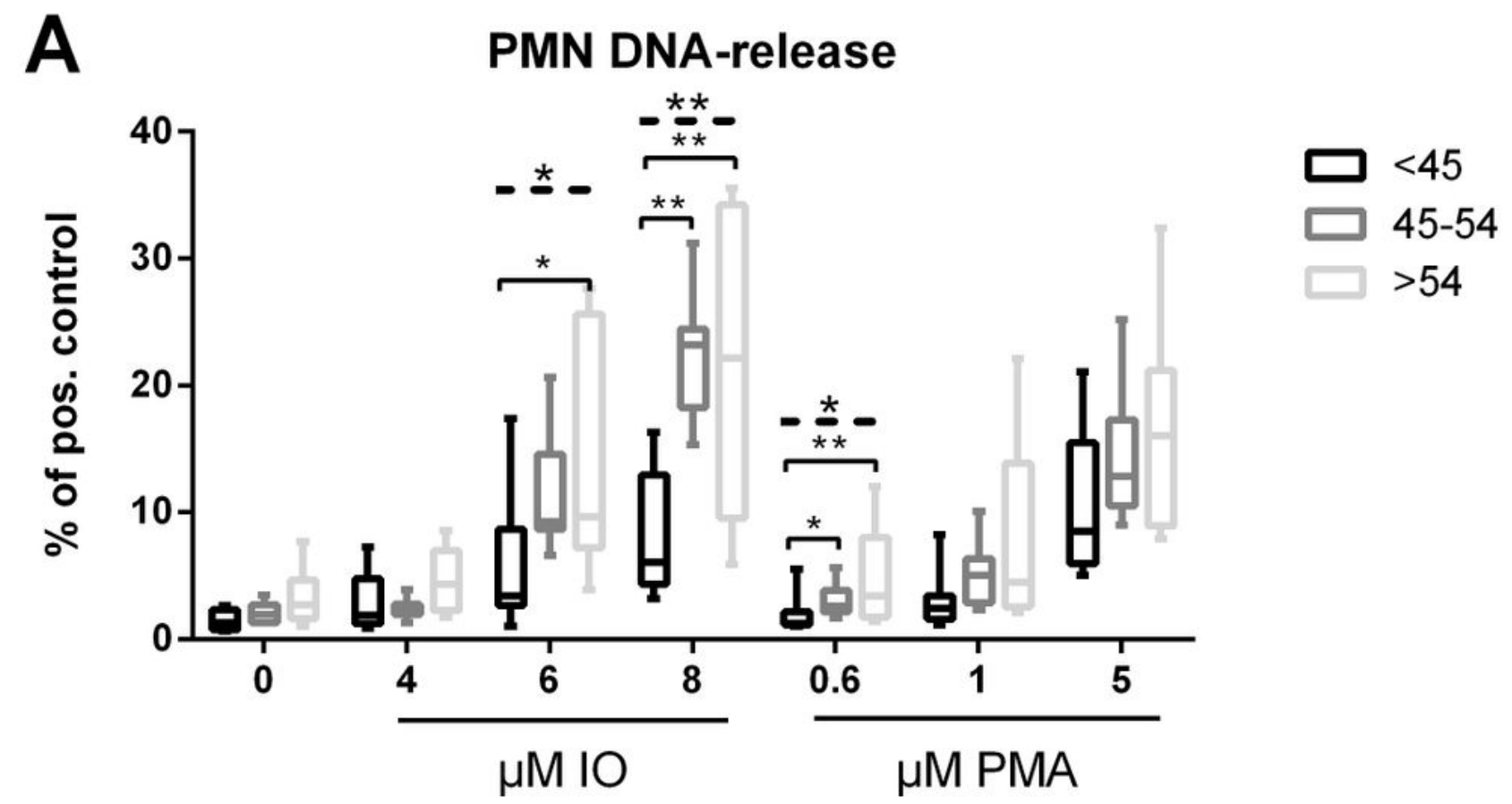

B

PBMC DNA-release

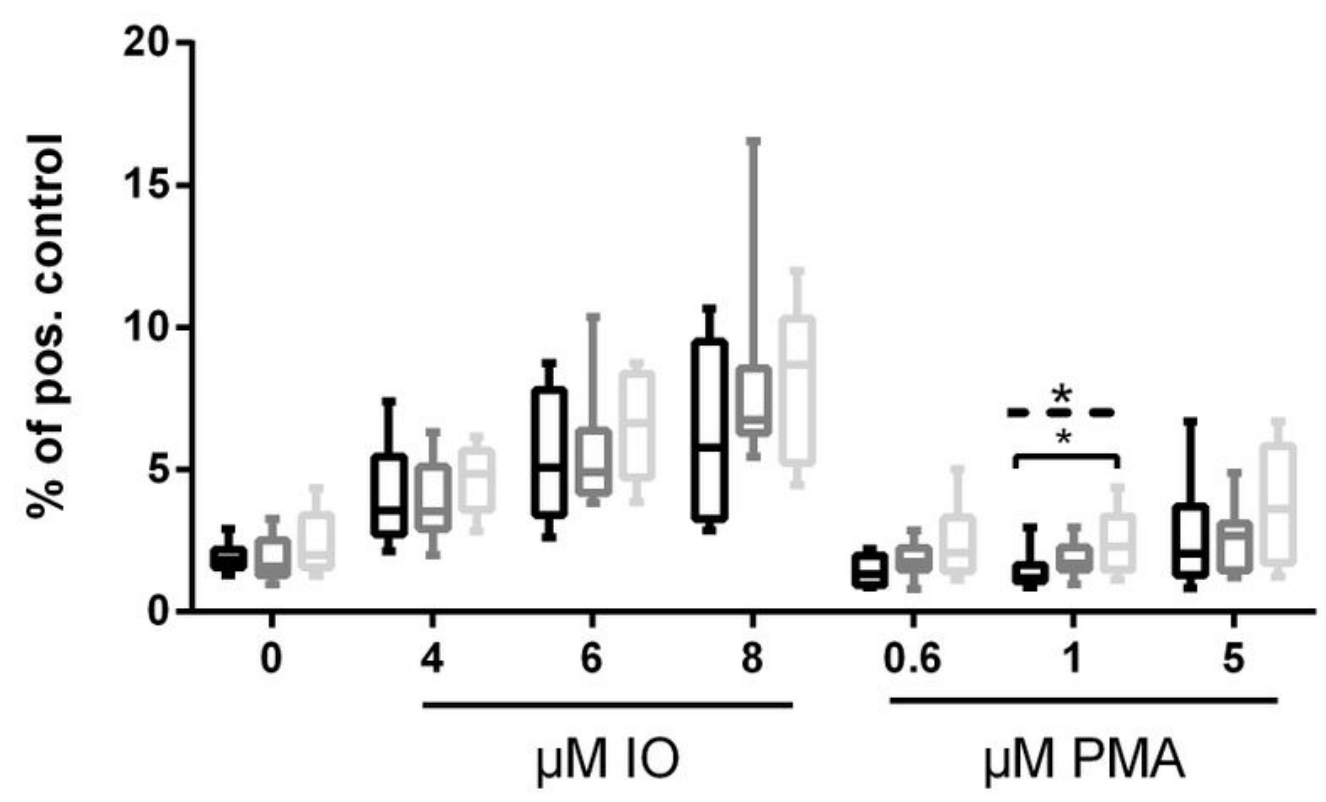

Figure 3

DNA-release of PMNs and PBMCs. Percentages of released DNA from (A) PMNs and (B) PBMCs compared to a positive control (cells treated with Triton-X 100). Unstimulated (0) as well as ionomycin (IO) and phorbol 12-myristate 13-acetate (PMA) treated PMNs show an increase in DNA release with increasing age. PMNs as well as PBMCs were stimulated for $2 \mathrm{~h} 45 \mathrm{~min}$ at $37^{\circ} \mathrm{C}$, Sytox Green was then 
added and samples incubated for $15 \mathrm{~min}$ at $37^{\circ} \mathrm{C}$ before measurement. p-values (Kruskal-Wallis test in dashed lines; post hoc test in solid lines): ${ }^{*} p \leq 0.05,{ }^{* *} p \leq 0.01$

\section{Figure 4}

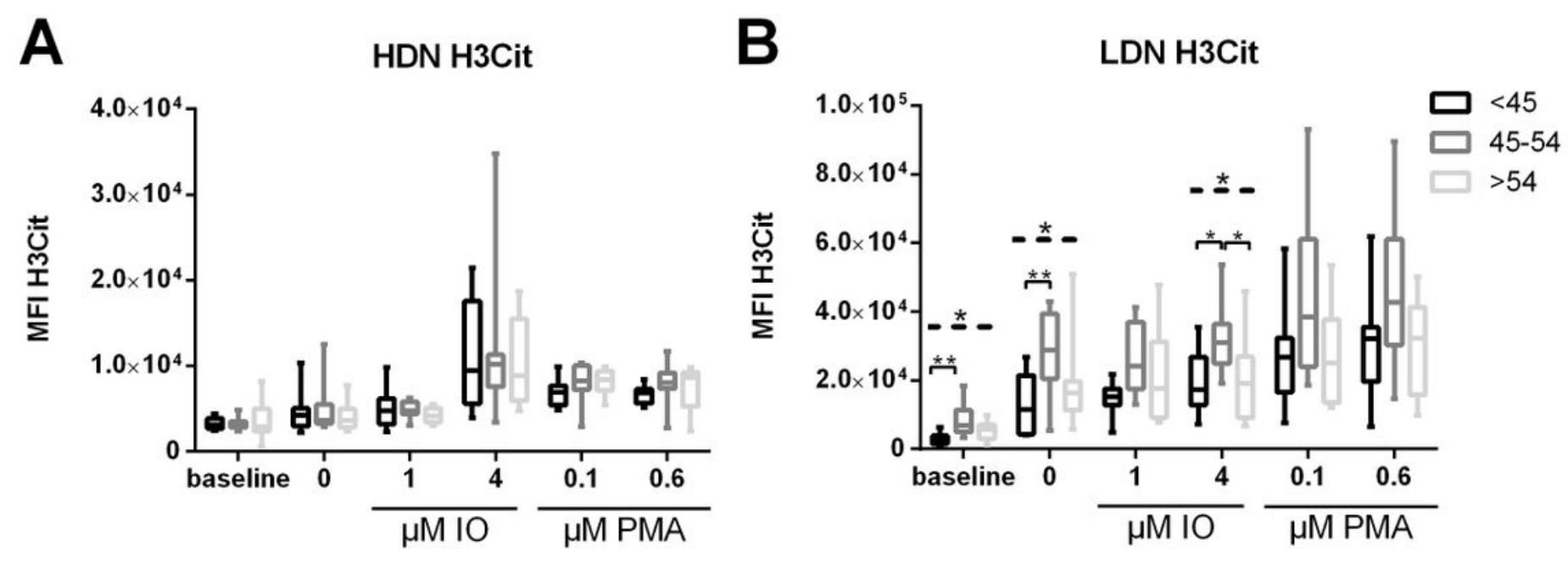

Figure 4

Levels of H3Cit in 3h stimulated HDNs and LDNs. (A) Median fluorescent intensity (MFI) of citrullinated histone $\mathrm{H} 3$ (H3Cit) positive baseline, unstimulated (0) as well as $3 \mathrm{~h} 10$ and PMA treated HDNs and (B) LDNs. $p$-values (Kruskal-Wallis test in dashed lines; post hoc test in solid lines): ${ }^{*} \leq \leq 0.05,{ }^{\star \star} p \leq 0.01$

\section{Supplementary Files}

This is a list of supplementary files associated with this preprint. Click to download.

- Sl.docx 